\title{
Prevalence Des Infections Parasitaires Dues Aux Protozoaires Identifies Au Laboratoire National De Biologie Clinique Et De Sante Publique, Bangui Republique Centrafricaine
}

\author{
Ernest Lango-Yaya
}

Laboratoire National de Biologie Clinique et de Santé Publique, Ministère de la Santé Publique, et de la Population, Bangui, République Centrafricaine

Faculté des Sciences de la Santé, Université de Bangui, République Centrafricaine

\section{Tatiana Ngalema}

Freddy Marcelin Agboko

Romaric Lebon Bondom

Marcellin Namzeka

Laboratoire National de Biologie Clinique et de Santé Publique, Ministère de la Santé Publique, et de la Population, Bangui, République Centrafricaine

\section{Donatien Clotaire Rafai}

Laboratoire National de Biologie Clinique et de Santé Publique, Ministère de la Santé Publique, et de la Population, Bangui, République Centrafricaine

Faculté des Sciences de la Santé, Université de Bangui, République

\section{Centrafricaine}

\section{Doi:10.19044/esj.2021.v17n21p115}

Submitted: 02 January 2021

Accepted: 24 May 2021

Published: 30 June 2021
Copyright 2021 Author(s)

Under Creative Commons BY-NC-ND 4.0 OPEN ACCESS

Cite As:

Lango-Yaya E., Ngalema T., Agboko F.M., Bondom R.L, Namzeka M. \& Rafai D.C. (2021). Prevalence Des Infections Parasitaires Dues Aux Protozoaires Identifies Au Laboratoire National De Biologie Clinique Et De Sante Publique, Bangui Republique Centrafricaine. European Scientific Journal, ESJ, 17(21), 115.

https://doi.org/10.19044/esj.2021.v17n21p115

\section{Résumé}

Les Protozoaires sont des parasites de petite taille, de forme diverses parmi lesquels on distingue les Protozoaires intestinaux (Amibes, Flagellés, Coccidies, Ciliés et Microsporidies) qui sont les plus répandus. Selon l’OMS, les parasitoses intestinales constituent un problème de santé publique dans le monde en général et en Afrique en particulier. Les formes graves sont 
généralement provoquées par certains protozoaires intestinaux à savoir Entamoeba histolytica, Giardia intestinalis et Trichomonas intestinalis qui jouent un rôle dans la survenue du syndrome dysentérique. C’est dans ce contexte que cette étude est menée dans l'objectif est d'évaluer la prévalence des Protozoaires digestifs selon leur degré de pathogénicité chez les patients. La mise en évidence des parasites a été effectuée par la technique de Formolether et de l'examen à l'état frais. Cette étude a montré que sur 11500 patients, 3922 soit 34,1\% étaient parasités. Parmi les patients parasités le sexe féminin était prédominant avec un pourcentage de $57,51 \%$. La tranche d'âge la plus touchée est celle comprises entre 20 à 29 ans (39,4\%), les enfants de moins de 1 an étaient moins touchés (1,2\%). Le parasite le plus représentatif est le kyste d'Entamoeba histolytica 30,40\% suivi de Trichomonas intestinalis 1,4\%. L'amibiase, la giardiose et la trichomonose restent les protozooses les plus pathogènes associées à l'hygiène défectueuse et du péril fécal.

Mots clés: Prévalence, Protozoaires, Entamoeba, Giardia, Trichomonas

\section{Prevalence Of Parasitic Infections Due To Protozoa Identified At The National Laboratory Of Clinical Biology And Public Health, Bangui Central African Republic}

\section{Ernest Lango-Yaya}

Laboratoire National de Biologie Clinique et de Santé Publique, Ministère de la Santé Publique, et de la Population, Bangui, République Centrafricaine

Faculté des Sciences de la Santé, Université de Bangui, République Centrafricaine

\section{Tatiana Ngalema}

Freddy Marcelin Agboko

Romaric Lebon Bondom

\section{Marcellin Namzeka}

Laboratoire National de Biologie Clinique et de Santé Publique, Ministère de la Santé Publique, et de la Population, Bangui, République Centrafricaine

\section{Donatien Clotaire Rafai}

Laboratoire National de Biologie Clinique et de Santé Publique, Ministère de la Santé Publique, et de la Population, Bangui, République Centrafricaine

Faculté des Sciences de la Santé, Université de Bangui, République Centrafricaine

\section{Abstract}

Protozoa are parasites of small size, of various shapes, among which we distinguish the intestinal Protozoa (Amoeba, Flagellates, Coccidia, Ciliates 
and Microsporidia) which are the most widespread. According to WHO, intestinal parasitosis is a public health problem in the world in general and in Africa in particular. The severe forms are generally caused by certain intestinal protozoa namely Entamoeba histolytica, Giardia intestinalis and Trichomonas intestinalis which play a role in the occurrence of dysenteric syndrome. It is in this context that this study is being carried out with the objective of assessing the prevalence of digestive protozoa according to their degree of pathogenicity in patients. The detection of the parasites was carried out by the formalin-ether technique and the examination in the fresh state. This study showed that out of 11,500 patients, 3,922 or $34.1 \%$ were parasitized. Among the parasitized patients the female sex was predominant with a percentage of $57.51 \%$. The most affected age group is between 20 to 29 years old (39.4\%), children under 1 year old were less affected (1.2\%). The most representative parasite is the Entamoeba histolytica cyst $30.40 \%$ followed by Trichomonas intestinalis $1.4 \%$. Amebiasis, giardiasis and trichomoniasis remain the most pathogenic protozoa associated with poor hygiene and faecal hazard.

Keywords: Prevalence, Protozoa, Entamoeba, Giardia, Trichomonas

\section{Introduction}

Les protozoaires sont des parasites de petite taille et de forme diverses parmi lesquels les Protozoaires intestinaux (Amibes, Flagellés, Coccidies, Ciliés et Microsporidies). Ces espèces peuvent provoquer des protozooses intestinales plus ou moins grave. Il s'agit particulièrement de l'amibiase, la giardiose et la cryptosporidiose (Coudert et al., 2010). Très largement répondus à travers le monde, les parasitoses intestinales constituent un sérieux problème de santé publique. Il a été estimé que plus de 3 milliards de personnes sont infectées par les parasitoses intestinales dans le monde (Thellier et al., 2007). Le tube digestif de l'être humain peut être colonisé par diverses espèces parasites. Qu'il s'agisse de Protozooses ou d'Helminthiases, ces parasitoses digestives siègent préférentiellement dans l'intestin. Cette situation stratégique au sein de l'hôte apporte au parasite un substrat nutritionnel régulier et assure la pérennité de son cycle de transmission. Majoritairement liées au péril fécal, seules certaines parasitoses intestinales peuvent être pathogènes pour l'organisme humain. Leur pathogénicité se traduit par l'agression de la paroi colique conduisant à colites asymptomatiques, voire des pseudotumeurs ; ce qui justifie leur prise en charge thérapeutique (Nicolas et al., 2001), (Rey et al., 2005). Après ingestion par l'homme, les oocystes perdent leur coque protectrice dans le tube digestif et libèrent des sporozoïtes, qui envahissent les cellules épithéliales de l'intestin grêle et causent de la diarrhée, des crampes abdominales ou des ballonnements et des nausées (Ortega et al., 2010) En effet Selon l’Organisation Mondiale de 
la Santé, plus de 2 millions de personnes, surtout des enfants de moins de cinq ans des pays en voie de développement meurent chaque année des maladies diarrhéiques du fait de l'insuffisance des mesures d'hygiènes et d'assainissement (OMS, 2014). Les parasitoses intestinales, bien qu'elles suscitent de nos jours peu d'intérêt à côté des maladies comme le SIDA, la tuberculose, le paludisme et l'onchocercose, elles constituent en milieu tropical un problème de santé publique, en raison essentielle des conditions climatiques favorables, de l'absence ou de l'insuffisance des mesures d'hygiène, d'assainissement et de pauvreté. En Afrique les facteurs tels que la promiscuité, le manque d'eau potable, l'hygiène alimentaire et l'insuffisance d'installation sanitaire ont fait que la prévalence globale des parasitoses intestinales était de 63,3\%, dont la majorité (53\%) était transmise par l'eau sale (Ohouya, 2015). La croissance démographique, l’immigration, les conditions climatiques, le faible niveau socio-économique et l'hygiène précaire sont des facteurs favorables pour l'extension du parasitisme dans une population (Afriad, 2018). Ces parasitoses sont souvent sévères chez les enfants, avec comme conséquence la malnutrition, l'anémie, la faible résistance à l'infection et un fort taux de mortalité infantile (Bouree, 2000). En République Centrafricaine $\{\mathrm{RCA}\}$, les parasitoses intestinales posent un problème de santé publique au sein de la population en raison des multiples crises qu'a connues le pays. Celles-ci ont engendré des multiples déplacements avec un manque d'eau potable, manque d'hygiène alimentaire et l'insuffisance d'installation sanitaire. Cette étude a pour objectif de de contribuer à une analyse épidémiologique des parasitoses au sein de la population selon leur degré de pathogénicité.

\section{Méthodologie \\ Cadre d'étude}

Cette étude a été réalisée au Laboratoire National de Biologie Clinique et de Santé Publique, Il s'agissait d'une étude rétrospective à viser descriptive enrôlant tous les échantillons des selles en provenance des 8 (huit) Arrondissements de la ville de Bangui, Bimbo et Begoua. Elle était basée sur l'analyse épidémiologique des parasitoses au sein de la population durant l'année 2017 à 2019 suite à une Infestation due aux protozoaires : à propos de 3922 soit 34,1\% parasités. L'analyse bactériologique de l'eau à l'alentour de ces zones a été également mise aux points.

\section{Analyse parasitologique des selles}

-Prélèvement des selles

Une quantité suffisante des selles a été prélevée et déposée dans un pot stérile, sur lequel est collée une étiquette portant l'identité du patient. Les 
selles ne devraient pas être mélangées avec des urines, du papier hygiénique ou des fragments de coton. Les selles sont amenées au Laboratoire.

\section{-Examen macroscopique}

La couleur et la consistance des selles à analyser ont été notées, ainsi que la présence éventuelle du sang, de glaires, des mucosités et du pus.

\section{-Examen direct}

Une partie de selle (5 mg) a été prélevée et étalée dans une goutte de sérum physiologique sur une lame et recouverte d'une lamelle. La préparation doit être mince et examinée au microscope à l'objectif x10. Une fois repérés, les parasites sont confirmés à l'objectif x40.

-Techniques d'enrichissement

\section{Concentration parasitaire au Formol-ether}

Un morceau de selles (2 g) a été écrasé et mélangé à $10 \mathrm{ml}$ de soluté physiologique. La suspension a été filtrée à travers 2 couches de Gaze dans un tube à centrifuger portant des graduations de $10 \mathrm{ml}$ et de $13 \mathrm{ml}$. Elle a été ensuite centrifugée pendant 1 minute à une vitesse moyenne. Le surnageant a été rejeté. Le culot a été lavé deux fois avec du soluté physiologique, ensuite $10 \mathrm{ml}$ de formol à $10 \%$ ont été ajoutés. Puis, le culot a été bien délayé et et laissé au repos pendant 5 minutes. $3 \mathrm{ml}$ d'éther ont été ajoutés (jusqu'à la graduation $13 \mathrm{ml}$ ). Le tube a été fermé et centrifugé pendant 1 minute à une vitesse réduite. A la fin de la centrifugation le tube contenait 4 couches: la première couche était de l'éther; la deuxième couche était des débris; la troisième couche était le formol et la quatrième couche était le culot, qui pouvait contenir des œufs et des kystes de parasites.

La deuxième couche a été décollée à l'aide d'un applicateur en bois, en glissant celui-ci entre la couche et les parois du tube, et le surnageant rejeté. A l'aide d'un écouvillon-coton, tous les débris adhérant encore à l'intérieur du tube ont été raclés. Bien mélanger le culot restant en tapotant doucement le fond du tube puis déposer sur une lame 2 gouttes de culot, l'une à une extrémité de la lame et l'autre à l'autre extrémité de la lame. Une goutte de lugol a été ajoutée uniquement à la deuxième goutte de culot. Les deux préparations ont été recouvertes de lamelles. La première préparation (non colorée) a été observée aux objectifs x10 et x40 afin d'observer les œufs et les larves. La deuxième préparation colorée a été seulement observée l'objectif x40 pour observer les kystes

\section{Analyses statistiques}

Les données recueillies ont été analysées à l'aide de Logiciels EXCEL 2010 et Epi info. Des tests statistiques tels que les tests Chi 2 ont aussi été utilisés pour les comparaisons avec un ddl de 0,005. 


\section{Résultats}

Tableau I. Caractéristiques sociodémographiques de la population d'études et Prévalence de contamination (\%)

\begin{tabular}{|l|l|l|l|}
\hline Variables & Effectifs & $\begin{array}{l}\text { Pourcentage } \\
(\mathbf{\%})\end{array}$ & $\begin{array}{l}\text { Prévalence de } \\
\text { contamination (\%) }\end{array}$ \\
\hline Sexe & & & - \\
\hline Masculin & 4874 & 42,4 & \\
\hline Féminin & 6626 & 57,6 & - \\
\hline Tranche d'âges (an) & & & \\
\hline$<\mathbf{1}$ & 141 & 1,2 & $24,99 \%$ \\
\hline $\mathbf{1 - 9}$ & 1676 & 14,6 & $35,55 \%$ \\
\hline $\mathbf{1 0 - 1 9}$ & 1219 & 10,6 & $18,88 \%$ \\
\hline $\mathbf{2 0 - 2 9}$ & 4533 & 39,4 & 12,54 \\
\hline $\mathbf{3 0 - 3 9}$ & 2009 & 17,5 & 10,5 \\
\hline $\mathbf{4 0 - 4 9}$ & 780 & 6,8 & 5,64 \\
\hline$\geq \mathbf{5 0}$ & 1142 & 9,9 & 2 \\
\hline
\end{tabular}

Le tableau 1 montre que le sexe féminin est plus représentatif que le sexe masculin avec un pourcentage respectivement 57,6 et $42,4 \%$. La tranche d'âge de 20 à 29 ans a une prévalence de 39,4\% et celle de 30 à 39 ans est de $17,5 \%$. Les taux d'infestation en fonction des tranches d'âge sont élevés dans la tranche d'âge de 1 à 9 ans (35,55\%), et la moins affectée de 10 à 19 ans (18,88\%). La tranche d'âge inférieure à 1 an dispose d'un taux de prévalence de 24,99\% relativement proche de ceux de 10 à 19 ans. En fait, la tranche d'âge de 1 à 9 ans exempte de toute activité, devrait courir le moins de risque de contamination. Malgré ces variations entre les tranches d'âge, les taux de prévalences diminue considérablement chez les adultes.

Tableau I. Répartition selon les patients parasités

\begin{tabular}{|l|l|l|}
\hline Patients & Effectifs & Pourcentage(\%) \\
\hline Parasités & 3922 & 34.1 \\
\hline Non parasités & 7578 & 65.9 \\
\hline Total & 11500 & 100 \\
\hline
\end{tabular}

Le Tableau II montre que sur un total de 11500 patients reçus pendant la période d'étude, 3922 étaient parasités soit un pourcentage de 34,1\%. La contamination était due à la qualité de l'eau consommée. Presque tous les puits sont contaminés par les germes. L'homme se contamine en consommant de l'eau non traitée ou en ingérant des aliments contaminés. 


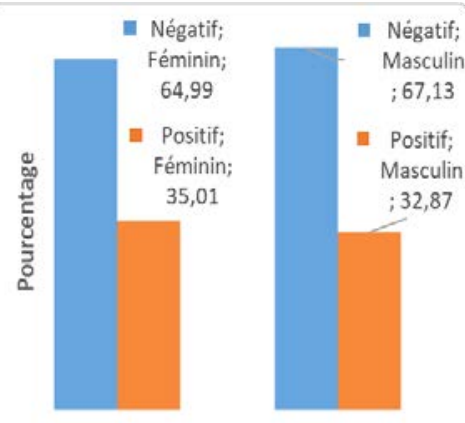

Figure I. Répartition des cas positif et négatif selon le sexe

Selon la figure 1, les femmes sont plus parasitées que les hommes. Le nombre de cas positif était de 2320 chez les patientes femmes et de 1602 chez les hommes. L'absence d'hygiène féco-orale, le manque des latrines aménagées, les mauvaises méthodes de conservation des aliments favorisent la multiplication des parasites dans les familles et dans les collectivités. Toutefois, il faut également noter que la prévalence des protozoaires dépendrait plus de la période d'étude. La contamination serait plus élevée en saison pluvieuse qu'en saison sèche.

Tableau IIII. Distribution de données selon l'année par rapport aux types de parasites

\begin{tabular}{|c|c|c|c|c|}
\hline & \multicolumn{3}{|c|}{ ANNEE } & \\
\hline Parasites & $\mathbf{2 0 1 7}$ & $\mathbf{2 0 1 8}$ & $\mathbf{2 0 1 9}$ & Total \\
\hline EHH & 44 & 40 & 24 & 108 \\
\hline Col\% & $1.28 \%$ & $0.97 \%$ & $0.61 \%$ & $0.94 \%$ \\
\hline EHH + TRICHO & 0 & 0 & 7 & 7 \\
\hline Col\% & $0.00 \%$ & $0.00 \%$ & $0.18 \%$ & $0.06 \%$ \\
\hline EHH & 1096 & 1392 & 1004 & 3492 \\
\hline Col\% & $31.93 \%$ & $33.90 \%$ & $25.34 \%$ & $30.37 \%$ \\
\hline KEH + EHH & 0 & 15 & 1 & 16 \\
\hline Col\% & $0.00 \%$ & $0.37 \%$ & $0.03 \%$ & $0.14 \%$ \\
\hline KEH + KGL & 10 & 5 & 2 & 17 \\
\hline Col\% & $0.29 \%$ & $0.12 \%$ & $0.05 \%$ & $0.15 \%$ \\
\hline KGL & 80 & 19 & 18 & 117 \\
\hline Col\% & $2.33 \%$ & $0.46 \%$ & $0.45 \%$ & $1.02 \%$ \\
\hline KEH & 2115 & 2613 & 2850 & 7578 \\
\hline Col\% & $61.63 \%$ & $63.64 \%$ & $71.93 \%$ & $65.90 \%$ \\
\hline TRICHO & 85 & 17 & 55 & 157 \\
\hline Col\% & $2.48 \%$ & $0.41 \%$ & $1.39 \%$ & $1.37 \%$ \\
\hline TOTAL & 3432 & 4106 & 3962 & 11500 \\
\hline Col\% & $100.00 \%$ & $100.00 \%$ & $100.00 \%$ & $100.00 \%$ \\
\hline
\end{tabular}




\begin{tabular}{|l|l|l|}
\hline $\mathbf{C h i}^{2}$ & df & Probabilité \\
\hline $\mathbf{2 8 9 . 8 9 4 4}$ & 18 & 0 \\
\hline
\end{tabular}

EHH = Entamoebahistolyticahistolytica $; \mathrm{KEH}=$ kysted'Entamoebahistolytica ; KGL= Kyste de Giardia lamblia ; TRICHO = Trichomonasintestinalis

L'analyse des registres du laboratoire National a montré que les types de parasites retrouvés sur cette période de deux ans ont été : Entamoeba histolytica histolytica; Kyste d'Entamoeba histolytica ; Kyste de Giardia lamblia et Trichomonas intestinalis. Les protozoaires les plus communément retrouvés étaient Entamoeba histolytica (30,37\%), Thricomonas intestinalis $(1,37 \%)$ et Gardia intestinalis (1,02 \%). La répartition des cas positif et négatif selon le sexe est représentée par la figure 1 . La prévalence des parasites intestinaux en année d'étude, était de 35,55 \% pour la tranche d'âge de 1 à 9 ans (tableau 1). Pour les tranches d'âge des adultes, on note une baisse significative de la prévalence, jusqu'à un minimum de $2 \%$. Le parasite le plus représentatif est le kyste d'Entamoeba histolytica (KEH) avec 65,90“\% suivi de forme végétative d'Entamoeba histolytica histolytica avec 30,37“\%. Le parasite le moins représenté est le Kyste de Giardia lamblia (1,02\%).

\section{Discussion}

Cette étude à viser descriptive transversale du type rétrospective, réalisée au Laboratoire National de Biologie Clinique et de Santé Publique de Bangui, montre que les parasitoses constituent un problème de santé publique à Bangui et ses environs. Durant la période de 2017 à 2019, sur les 11500 patients recensés, 3622 patients étaient parasités soit une prévalence de 34,1\%. Ce résultat est sensiblement proche de celle de (Ntumba Kabongo, 2012) qui a obtenu une prévalence de 31,9\% chez les enfants de 0 à 10 ans hospitalisés et examinés à l'hôpital de Kisanga en (RDC) et similaire au résultat de Diouf et al en 2020. La prévalence dans une étude réalisée par (Ndiaye, 2006) au Sénégal était de $54,4 \%$, cette prévalence est supérieure à celle de cette étude, cela pourrait s'expliquer par les différents modes et facteurs de contamination qui dépendent d'un pays à un autre. Selon certaines études, ces parasitoses comptent parmi les dix infections les plus répandues au monde (Ndiaye, 2006). En effet, plusieurs études ont établi l'existence de corrélations entre l'augmentation des concentrations de microorganismes et une turbidité accrue (Snead et al., 2011; Goshko et al., 2011; Haas et al., 2011). La qualité de l'eau et de la nourriture devrait donc être une priorité, le plus grand nombre des parasitoses fait suite à l'abondance des pluies (Mweze, 2003). Les eaux de puits ont contenu moins d'oocystes que les eaux de sources et de cours d'eau (Ngakomo-Ananga et al. 2020). La réduction de la turbidité permet l'inactivation des microorganismes surtout les oocystes de Cryptosporidium. Du point de vue sociodémographique, les femmes se sont présentées à la consultation plus que les hommes avec un pourcentage de 57,6 et 42,4\% 
respectivement. Ces résultats sont en adéquation avec ceux obtenus par Ndiaye, un pourcentage de $60,4 \%$ pour les femmes et $54,4 \%$ pour les hommes au centre de santé de Guédiawaye (Sénégal) en 2006. Le nombre de cas positif est plus élevé chez les patients de sexe féminin que ceux du sexe masculin. La tranche d'âge la plus affectée est celle de 20 à 29 ans suivie de celle de 30 à 39 ans ainsi. Les enfants de moins de 1 an sont moins représentés. Ces résultats sont conformes à ceux obtenus par (Nimo Ngbabo, 2008) dans une étude réalisée sur la prévalence des parasitoses intestinales à Kisangani qui a montré que la fréquence d'infestation des adultes est plus élevée que celle des enfants, soit 63,0\% contre 57,0\% (Nimo Ngbabo, 2008). L'étude a montré que la prévalence de kyste d'Entamoeba histolytica était de 30,4\%, suivi de Trichomonas intestinalis (1,4\%). Dans plusieurs études menées, l'amibiase était toujours plus représentative que les autres protozoaires. Ndiaye en 2006 avait trouvé une prévalence de 23,8\% pour Entamoeba histolytica, 20,8\% pour Giardia lamblia et $0,50 \%$ pour Trichomonas intestinalis. La forte prévalence de Entamoeba histolytica et de la Giardia lamblia serait liée à leur cycle de vie, ces parasites peuvent passer d'un état végétatif à un état kystique et vis versa. Il est à noter que les hommes sont plus parasités que les femmes que pour la co-infection Entamoeba histolytica plus Trichomonas intestinalis. Cependant en 2017 le nombre de cas positif était plus élevé $(38,4 \%)$ qu'en 2018 (36,4\%) et en 2019 (28,1\%).

\section{Conclusion}

Cette étude menée au Laboratoire National de Biologie Clinique et de Santé Publique sur les parasitoses intestinales, s'était intéressée sur les protozoaires en particulier constitue un problème de Santé Publique. Vu la prévalence de l'infestation obtenue dans cette étude, les femmes sont plus infestées que les hommes et de même, les adultes sont plus infestés que les enfants. La prévalence des infestations due à l'Entamoeba histolytica était plus élevée que celle de Giardia lamblia et de Trichomonas intestinalis. Les taux d'infestations obtenus sont liés à des notions élémentaires d'hygiène, des fosses septiques, des latrines très mal entretenues et de l'insalubrité d'environnement favorisant beaucoup plus l'infestation parmi la population d'étude.

\section{References:}

1. Coudert. P, Dreyfuss, (2010). G. Biologie et cycles parasitaires. Actualités pharmaceutiques. 500:18-22.

2. Thellier. M, Faussart. A, Danis. M, (2007). Epidémiologie mondiale des maladies parasitaires. La revue du Praticien. 57 : 131-136.

3. Nicolas. X, Chevalier. B, Simon. F, Klotz. F, "Traitement des parasitoses intestinales (amibiase et mycose excluses)", Encycl. Méd. Chir. (Elsevier, Paris), 9-062-A-60, pp. 1-13, 2001 [2] 
4. Rey. P, Andriamanantena. D, Bredin. C, Klotz. F, "Colites parasitaires", Encycl. Méd. Chir. (Elsevier, Paris), 9-062-A-45, pp. 19, 2005.

5. Ortega, YR., Sanchez, R. (2010). Update on Cyclospora cayetanensis, food-borne and waterborne parasite. Clin Microbiol Rev; 23(1):21834.

6. (OMS, 2014). Eau, Hygiène, Assainissement et Santé dans les quartiers précaires à Nouakchot (Mouritanie): Contribution à l'approche écosanté à tysaken https://doi.org/10.4000/vertigo.14999.

7. Ohouya. D.G, (2015). Prévalence des parasitoses intestinales chez les enfants de 0 à 5 dans la communauté d'anonkoi 3. Thèse de Pharmacie, Université Felix Houphouët Boigny, Abidjian Côte-d'Ivoire. p 149.

8. Afriad. Y, (2018). Epidémiologie des parasitoses intestinales chez la population de la ville 'd'Agadir. Thèse de doctorat en Médecine. Université CADI AYYAD, Marrakech, Maroc.

9. Bouree. P, (2000). Parasitose intestinal infantiles. Encyclopédie Médico-chirurgicale (Ed. Scientifique et Médicale EL Service SAS Paris, tout droit réservé). Pédiatrie 4-015-F-10, Akos, Encyclopédie Pratique de Médecine. 8- 1075.

10. Ntumba Kabongo. E, (2012). Prévalence des Parasitoses Intestinales chez les enfants de 0 à 10 ans de HGR Kisanga. UNILU-Licence en Santé Publique. Mémoire-Online Biologie-Médecine.

11. Diouf. S, Diallo. A, Camara. B, Diagne. I, Singate, SY. H, (2000). Parasitoses intestinales de l'enfant en zone rurale sénégalaise (KHOMBOLE). Médecine d'Afrique Noire. 47.

12. Ndiaye. A, (2006). Contribution à l'étude des parasitoses intestinales à l'institut de pédiatrie sociale de PIKINE GUEDIAWAYE. Thèse pour obtenir le grade de Docteur en Pharmacie (diplôme d'Etat). 78pages.

13. Snead, M.C., Olivieri, V.P., Kruse, C.W. et Kawata K. (2011). Benefits of maintaining a chlorine residual in water supply systems. U.S. Environmental Protection Agency, Washington, DC. Juin (EPA600/2-80-010).

14. Goshko, M.S., Minnigh, H.A., Pipes, W.O. et Christian, R.R. (2011). Relationships between standard plate counts and other parameters in water distribution systems. J. Am. Water Works Assoc., 75(11): 568571

15. Haas, C.N., Meyer, M.A. et Paller, M.S. (2011). Microbial alterations in water distribution systems and their relationship to physicalchemical characteristics. J. Am. Water Works Assoc., 75: 475-481. Review.

16. Mweze. N, (2003). Nature et fréquence des parasitoses intestinales, Monographie inédite UNIKIS, Fac de Méd. RDC. 
17. Ngakomo Ananga. R. P, Ajeagah. G. A, Elnaga. Z. A, Ngassam. P, (2020). Caractéristique Physico-Chimique Etdynamique des Formes Environnementales des Coccidies Entériques Dans Les Eaux De Sources, Puits et Cours D'eau dans La Commune d'Akono(Cameroun, Afrique Centrale). European Scientific Journal. 16(3) : 1857 - 7881.

18. Nimo Ngbabo. S, (2008). Prévalence des parasitoses intestinales au C.S. Boyoma/Kisangani UNIKIS - Graduat. RDC. 